\title{
Sex in unisexual salamanders: discovery of a new sperm donor with ancient affinities
}

\author{
JP Bogart ${ }^{1}$, J Bartoszek ${ }^{2}$, DWA Noble ${ }^{1}$ and $\mathrm{K} \mathrm{Bi}^{1}$ \\ ${ }^{1}$ Department of Integrative Biology, University of Guelph, Guelph, Ontario, Canada and ${ }^{2}$ Department of Biological Sciences, \\ Wright State University, Dayton, OH, USA
}

\begin{abstract}
Although bisexual reproduction has considerable evolutionary benefits, several all-female vertebrates exist. Unisexual salamanders in the genus Ambystoma are common around the Great Lakes region in eastern North America. They originated from a hybridization event that involved a female that shared a common ancestor with Ambystoma barbouri 2.4 to 3.9 million years ago but, unexpectedly, $A$. barbouri nuclear genomes were unknown in unisexuals. Unisexual salamanders steal sperm from donors of normally bisexual species, so their reproductive mode is described as kleptogenesis. Most known unisexuals are polyploid and they all possess at least one $A$. laterale genome. One or more other genomes are taken from sperm donors that may include $A$. jeffersonianum, $A$. laterale, $A$. texanum and A. tigrinum. We examined unisexual adults and larvae in a
\end{abstract}

southern Ohio pond where unisexual individuals coexist with male $A$. barbouri. This population provided an opportunity to test hypotheses pertaining to the role of $A$. barbouri in the evolution of the disparate cytoplasmic and nuclear genomes in unisexual salamanders. Microsatellite DNA loci, mitochondrial DNA sequences and genomic in situ hybridization were used to identify the genomic constitution of individuals. A. barbouri was found to be an acceptable sperm donor for unisexuals but only contributed genomes in ploidy-elevated individuals. In the absence of $A$. jeffersonianum, this Ohio population is likely experiencing a recent switch in sperm donors from $A$. jeffersonianum to $A$. barbouri and demonstrates the evolutionary flexibility and dynamics of kleptogenesis.

Heredity (2009) 103, 483-493; doi:10.1038/hdy.2009.83; published online 29 July 2009

Keywords: Ambystoma unisexuals; Ambystoma barbouri; kleptogenesis; microsatellites; GISH

\section{Introduction}

The existence and persistence of unisexual and asexual animals has been a conundrum for evolutionary biologists. The obvious fitness advantages of an all-female population, where every individual is capable of producing offspring, must be weighed against the expected fitness advantages of genetic variation that is generally attributed to bisexual reproduction (Williams, 1975; Maynard Smith, 1992). Thus, the evolutionary short-term gain that an all-female population might enjoy likely confers detrimental costs and, as a result, only a few asexual lineages have a long geological history (Normark et al., 2003). Unisexual vertebrates have independently evolved in several lineages of fish, amphibians and reptiles (Dawley and Bogart, 1989; Avise, 2008), and although these vertebrate lineages are made up of all, or mostly all females, they are not necessarily asexual. Reproductive modes used by unisexual vertebrates vary with respect to the role that males may play. Only unisexual reptiles are known as truly parthenogenetic vertebrates and do not require males. Nevertheless, even among the well-studied parthenogens in the lizard genus Aspidocelis (formerly Cnemidophorus), occasional crosses between parthenogenetic females and males of bisexual

Correspondence: Dr JP Bogart, Department of Integrative Biology, University of Guelph, 50 Stone Road East, Guelph, Ontario, Canada N1G 2W1.

E-mail: jbogart@uoguelph.ca

Received 11 February 2009; accepted 21 May 2009; published online 29 July 2009 species do occur (Manning et al., 2005) and triploid unisexual species of Aspidocelis have been derived from crosses involving unisexual diploid females and bisexual males (Dessauer and Cole, 1989). In gynogenesis, sperm from a sympatric bisexual male serves only to stimulate the development of unreduced eggs, which is genetically equivalent to parthenogenesis but is sperm dependent. Hybridogenesis is a hemiclonal reproductive mode where hybrid females produce reduced eggs that possess only the female's genome. A male's genome is incorporated in the offspring for one generation but is subsequently eliminated in meiosis.

Unisexual salamanders in the North American genus Ambystoma are a common component of many populations around the Great Lakes region where they coexist with, and incorporate genomes from, one of four sperm donors. The first recognized, and most widely distributed, sperm donors are Ambystoma jeffersonianum (JJ) and A. laterale (LL) (Uzzell, 1964; Bogart and Klemens, 1997, 2008). But, unisexuals also use A. texanum (TT) as a sperm donor in Illinois, Michigan and Ohio (Downs, 1978; Morris and Brandon, 1984; Kraus, 1985a), and on the Lake Erie Islands: Bass (Downs, 1978), Pelee (Bogart et al., 1985) and Kelleys (Bogart et al., 1987). Unisexuals in those populations are diploid (LT), triploid (LTT) and tetraploid (LJJT, LLTT; LTTT). A. tigrinum (TiTi) is used as a sperm donor in Indiana (Morris, 1985) and Ohio (Kraus, 1985b; Bogart et al., 1987; Bogart, 2003) where LJJTi, LTTi and LTTTi unisexuals exist. Males of these four, mostly allopatric, species serve as sperm donors for the persistence of unisexual Ambystoma in various parts 
of their range. Bogart et al. (2007) used microsatellite DNA loci to examine the reproductive mode used by unisexual Ambystoma. They tested and rejected hypotheses and premises of gynogenesis and hybridogenesis, and concluded that a better descriptor for the reproduction of these salamanders was kleptogenesis because females steal sperm from sympatric males of bisexual species for recruitment and persistence. The acquired sperm may only serve to stimulate unreduced eggs to develop (as in gynogenesis) but a male's genome can be incorporated to replace an existing genome or to increase the ploidy level in unisexual offspring (Bi et al., 2008b). Kleptogenesis has resulted in the establishment of diploid, triploid, tetraploid and pentaploid unisexuals possessing more than 20 different nuclear genomic combinations or biotypes (Bogart, 2003; Bogart unpublished).

Based on sequencing matrilineally inherited mitochondrial DNA ( $m t \mathrm{DNA}$ ) genes, unisexual Ambystoma had a single origin 2.4-3.9 million years ago and their $m t$ DNA sequences align most closely with individuals from a Kentucky population of A. barbouri (Bogart, 2003, Bogart et al., 2007). Paradoxically, A. barbouri has not been found in the nuclear genome of any known unisexual. Distinguishing between, or identifying, the genomes in the various biotypes has traditionally involved observing the differential mobility of allozymes at isozyme loci that are diagnostic for each of the species that may be included in a unisexual (Bogart et al., 1987; Bogart and Klemens, 1997). More recently, microsatellite DNA alleles at polymorphic loci (Julian et al., 2003, Bogart et al., 2007) and fluorescent cytogenetic techniques (Bi and Bogart, 2006) are used as nonlethal methods to identify individuals and biotypes.

While conducting surveys of salamanders in southern Ohio, one of us (J Bartoszek) surveyed a vernal pond that contained a large number of breeding ambystomatid salamanders. Most individuals were females that, based on the known occurrence of $A$. jeffersonianum in the region, were suspected to be bisexual $A$. jeffersonianum and A. laterale -2 jeffersonianum (LJJ) unisexuals. A few individuals, however, had a morphology that was consistent with $A$. barbouri, which is a stream-breeding species that is found in Ohio, Kentucky and Tennessee (Petranka, 1998) and was not expected to live in a vernal pond with unisexual individuals. This population provided a unique opportunity to examine the interaction of unisexuals living with the 'mother' of unisexuals. If $A$. jeffersonianum males and $A$. barbouri females can coexist and hybridize, this could mimic the hypothetical hybridization event of an $A$. barbouri-like female that possibly crossed with an A. laterale-like male more than two million years ago (Bogart, 2003; Bogart et al., 2007). Presently, $A$. laterale is only found in a few distant northern Ohio populations (Petranka, 1998), so any recent hybrids in this southern Ohio population would not be expected to have $A$. laterale genomes.

Although previous $m t$ DNA sequence-based phylogenies showed $A$. barbouri individuals from a Kentucky population are closest to a recent maternal ancestor of unisexual Ambystoma (Bogart et al., 2007), A. barbouri demonstrate considerable $m t \mathrm{DNA}$ sequence diversity over the range of stream-breeding individuals (Niedzwiecki, 2005). Perhaps A. barbouri individuals in this Ohio pond are genetically distinctive or phylo- genetically closer to the unisexual clade. Additionally, because all sampled unisexuals have $m t \mathrm{DNA}$ that is more closely related to $A$. barbouri than it is to any of the four known sperm donors, possible nuclear-cytoplasmic incompatibilities would be expected to be minimized for unisexuals that used $A$. barbouri as a sperm donor and the barbouri genome could be easily incorporated or replace non-barbouri genomes. If female unisexuals mate with $A$. barbouri, and if an $A$. barbouri genome replaces an $A$. laterale genome, those unisexuals would not possess an $A$. laterale genome. If $A$. jeffersonianum genomes were replaced, unisexuals would be LJB or LBB if the A. laterale genome was somehow protected. Genetic and cytogenetic analyses of individuals in this pond were conducted to identify the breeding population and to test these hypotheses.

\section{Materials and methods}

\section{Source of specimens}

The pond was located in Mitchell Memorial Forest (MMF), Hamilton County, in southwestern Ohio. Ambystomatid salamanders enter ponds very early in the spring to breed. At that time, large, modified minnow traps were used to obtain 115 adult individuals. Small (3 to $5 \mathrm{~mm}$ ) tail tip samples were taken from all collected adults and preserved in $70 \%$ ethanol in $1.5 \mathrm{ml} \mathrm{micro-}$ centrifuge tubes. A few adult individuals were saved for voucher specimens but most were released at their point of capture after the tail tip sample had been taken. Ten egg masses were collected that were found adhering to submerged branches in the MMF pond. They were transported to the laboratory in Guelph, Ontario for hatching and rearing. Hatched larvae were fed brine shrimp nauplii (Artemia sp.) and Blackworms (Aquatic Foods Ltd, Fresno, CA, USA) until they were large enough for small tail tips to be excised for DNA extractions and for chromosome analyses. Tail tip samples were also obtained from 10 adult individuals from another pond in southern Ohio, Fort Ancient (FA). FA was believed to be a breeding pond for $A$. jeffersonianum and did not contain unisexual individuals (J Bartoszek, personal observation).

\section{Microsatellite DNA}

Total genomic DNA was extracted from adult and larval tail tips using the protocol provided with a Promega Wizard Genomic DNA Purification kit. Extracted DNA was rehydrated in $100 \mu \mathrm{l}$ water and stored at $-20^{\circ} \mathrm{C}$ before being used for PCR amplification of microsatellite DNA alleles. Species primers are not available for A. laterale or for $A$. barbouri but primers designed for A. jeffersonianum often amplify microsatellite DNA alleles in other ambystomatids. Amplified products may have very different sizes in cross-species amplification so, for some loci, it is often possible to identify genome-specific microsatellite DNA alleles (Julian et al., 2003). Alleles were examined for six polymorphic, tetranucleotide microsatellite DNA loci. From a previous study (Bogart et al., 2007) we found that raising the PCR reaction volume from $10 \mu \mathrm{l}$ to $25.5 \mu \mathrm{l}$ provided more consistent results and alleviated problems related to template quantity and the unpredictable numbers of speciesspecific genomes in unisexual samples. The PCR reaction 
mix for each sample consisted of $1.5 \mu$ l of the hydrated DNA extraction, $1.0 \mu \mathrm{l}\left(10 \mathrm{pmol} \mu \mathrm{l}^{-1}\right)$ each of labeled forward and unlabeled reverse primers, $0.6 \mu \mathrm{l} \mathrm{dNTP}$ (10 mM of each dNTP: Roche Diagnostics, Mannheim, Germany), $0.15 \mu \mathrm{l}$ Taq polymerase (New England Biolabs, Ipswich, MA, USA), $2.5 \mu$ l homemade PCR Buffer (each $\mathrm{ml}$ consisted of $25 \mu \mathrm{l} 1 \mathrm{M} \mathrm{MgCl}, 100 \mu \mathrm{l} 1 \mathrm{M}$ Tris ( $\mathrm{pH} 8.3$ ), $500 \mu \mathrm{l} 1 \mathrm{M} \mathrm{KCl}, 80 \mu \mathrm{l}$ bovine serum albumin (10 $\mathrm{mg} \mathrm{ml}^{-1}$; Sigma, St Louis, MO, USA), $50 \mu \mathrm{l} 2 \%$ gelatin (Sigma), $245 \mu \mathrm{l}$ water) and $18.75 \mu \mathrm{l}$ water. The PCR reaction was initiated with a 1 min denaturation at $94{ }^{\circ} \mathrm{C}$ followed by 30 cycles of $94{ }^{\circ} \mathrm{C} /$ annealing temperature of $57^{\circ} \mathrm{C}$ (AjeD94, AjeD346, AjeD422, Atex74) or $58^{\circ} \mathrm{C}$ (AjeD283, AjeD378) $/ 72{ }^{\circ} \mathrm{C}$ extension. The primers and annealing temperatures for the AjeD loci were provided by Julian et al. (2003) and for Atex74 by Williams and Dewoody (2004). Locus AjeD378 amplifies A. jeffersonianum alleles but does not amplify $A$. laterale alleles. Loci AjeD94 and AjeD346 amplify multiple alleles in both A. jeffersonianum and $A$. laterale and have allelic size ranges with little overlap between those species. Locus Atex74 does not amplify either $A$. jeffersonianum or A. laterale alleles, but amplifies alleles in $A$. texanum (Williams and Dewoody, 2004) and A. barbouri (this study). Microsatellite alleles overlap in size in A. laterale and A. jeffersonianum for AjeD283 and AjeD422 but are highly variable, provide additional genotypic information and assist with ploidy determination. Forward primers for each locus were fluorescently labeled with tetramethyl rhodamine. PCR products were electrophoresed on vertical, $6 \%$ denaturing polyacrylamide gels alongside a Genescan-350 TAMRA size standard ladder. Gels were scanned with a Hitachi FMBioII imager and were scored relative to the ladder using Hitachi FMBioII imaging software version 1.5. Scoring was verified visually to ensure accuracy. PCR reactions of the same samples were repeated, and the position of the samples on the gel was changed to minimize possible scoring errors. Ploidy was assigned to an individual based on the largest number of microsatellite DNA alleles observed at any locus and the lowest number of genomes that could be present based on genome specific microsatellite DNA alleles.

\section{Mitochondrial DNA sequences}

We selected DNA extractions from representative individuals based on the multilocus genotypes obtained in the microsatellite analyses to sequence a portion of the $m t$ DNA genome to identify unisexual and bisexual individuals in the pond. Primers MLM-THR and MLM651 (Shaffer and McKnight, 1996) were used to amplify the entire D-loop, intergenic spacer region, $t_{R N A}{ }^{\text {Pro }}$, $\mathrm{tRNA}^{\text {Phe }}$ and part of the $\mathrm{tRNA}^{\mathrm{Thr}}$ genes. The PCR procedure was the same as used by Shaffer and McKnight (1996) with the annealing temperature being optimized at $46^{\circ} \mathrm{C}$. The sequences were aligned using CLUSTAL $X$ (Thompson et al., 1994) and compared with haplotypes recovered from other unisexual and bisexual individuals by Bogart et al. (2007) that used sequences from this same $>1 \mathrm{~kb}$ region of the mitochondrial genome.

\section{Chromosomes}

An in vitro saline-colchicine incubation of larval tail tips method of chromosome preparation followed procedures outlined by $\mathrm{Bi}$ and Bogart (2006). Chromosome squash preparations were observed and suitable spreads were photographed with phase contrast optics before removing the cover glass with liquid nitrogen. Chromosomes were difficult to obtain from some larvae that were not growing rapidly. If suitable chromosomes were not obtained initially, a second attempt, using regenerating tail tissue, was usually more successful than the primary tail tip sample. Slides that contained adequate metaphase spreads with few overlapping chromosomes were dehydrated in 95\% ethanol, stored at room temperature for 2-4 days and then maintained at $-20^{\circ} \mathrm{C}$ for genomic in situ hybridization (GISH) experiments using procedures described by $\mathrm{Bi}$ and Bogart (2006) but modified to include labeled probes for both $A$. laterale and $A$. barbouri and blocking DNA from $A$. jeffersonianum and $A$. texanum. As unisexual individuals are known to use $A$. texanum, the species most closely related to $A$. barbouri, as a sperm donor and some unisexuals do possess genotypes that include $A$. texanum in Ohio, blocking DNA from $A$. texanum was included to identify, if present, $A$. barbouri chromosomes and not A. texanum chromosomes. Probe and blocking DNA were obtained from specimens that were identified using isozymes and $m t \mathrm{DNA}$ sequences. Total genomic DNA from $A$. barbouri and $A$. laterale was labeled with biotin and DIG-nick translation kits (Roche) respectively. The probe mixture consisted of $5 \mathrm{ng}^{-1}$ biotin-labeled $A$. barbouri genomic probes and $5 \mathrm{ng}^{-1} \mathrm{l}^{-1}$ digoxigeninlabeled $A$. laterale genomic probes and approximately $75-100 \mathrm{ng} \mathrm{l}^{-1}$ A. jeffersonianum and $A$. texanum blocking DNA in $2 \times$ SSC (VWR, West Chester, PA, USA), $50 \%$ deionized formamide (Fisher Scientific, Ottawa, ON, Canada), $10 \%$ dextran sulfate salt (Sigma), $1 \times$ Denhardt's solution (Sigma) and 0.1\% SDS (Sigma). After hybridization and washing the slides, digoxigenin-labeled probes were detected with antidigoxigenin antibodies conjugated with fluorescein isothiocyanate (FITC; Roche), biotinylated probes were detected with rhodamine conjugated avidin DCS (Vector). The chromosomes were counterstained with 4',6-diamidino-2-phenylindole dihydrochloride (DAPI; Vector). After in situ hybridizations, chromosomes were observed using a Leica fluorescent microscope equipped with the appropriate filter sets for FITC (green), rhodamine (red) and DAPI (blue). The digital color images were recorded using a CCD camera and combined and analyzed with openlab 3.5.0.

\section{Results}

\section{Adult salamanders}

Microsatellite DNA alleles were obtained from all 115 adult individuals collected from the pond in MMF and from the 10 adults from FA (Table 1). Based on microsatellite DNA allele sizes reported by Julian et al. (2003) and Bogart et al. (2007), the majority of the MMF population (95 of 115) consisted of unisexual triploid A. laterale -2 jeffersonianum (LJJ) individuals. Seven diploid individuals possessed microsatellite alleles that amplified with primers for AjeD283, AjeD346, AjeD422 and Atex74. Based on a lack of amplified products using primers for AjeD378, these diploids did not possess A. jeffersonianum alleles. They were determined as A. barbouri based on morphology (Petranka, 1998) of the voucher specimens, and their $m t$ DNA haplotypes that were identical to $A$. barbouri haplotype E (compare 
Table 1 Genotypes found at six microsatellite loci from DNA extracted from adult salamander tail tip samples collected from a vernal pond in Mitchell Memorial Forest (MMF) and from a pond at Fort Ancient (FA), Hamilton County, Ohio

\begin{tabular}{|c|c|c|c|c|c|c|}
\hline & AjeD94 & AjeD283 & $\begin{array}{c}\text { Microsatellite locus } \\
\text { AjeD346 }\end{array}$ & AjeD378 & AjeD422 & Atex 74 \\
\hline \multicolumn{7}{|c|}{ MMF LJJ } \\
\hline (17) & $150 / 222 / 246$ & $138 / 154$ & $136 / 284$ & $220 / 256$ & $248 / 256 / 288$ & - \\
\hline (13) & $150 / 218 / 246$ & $138 / 154$ & $136 / 284$ & $220 / 256$ & $248 / 256 / 284$ & - \\
\hline (12) & $150 / 222 / 238$ & $134 / 154$ & $136 / 284$ & $220 / 256$ & $248 / 256 / 284$ & - \\
\hline (8) & $150 / 226 / 242$ & $134 / 154$ & $136 / 284$ & $220 / 256$ & $248 / 256 / 288$ & - \\
\hline (7) & $150 / 226 / 246$ & $134 / 154$ & $136 / 284$ & $220 / 256$ & $248 / 256 / 288$ & - \\
\hline (6) & $150 / 222 / 246$ & $134 / 154$ & $136 / 284$ & $220 / 256$ & $248 / 256 / 288$ & - \\
\hline (6) & $150 / 222 / 250$ & $138 / 154$ & $136 / 284$ & $220 / 256$ & $248 / 256 / 288$ & - \\
\hline (5) & $150 / 222 / 242$ & $134 / 154$ & $136 / 284$ & $220 / 256$ & $248 / 256 / 288$ & - \\
\hline (3) & $150 / 222 / 242$ & 134/154/158 & $136 / 284$ & $220 / 256$ & $248 / 256 / 284$ & - \\
\hline (3) & $150 / 218 / 246$ & $138 / 154 / 158$ & $136 / 284$ & $220 / 256$ & $248 / 256 / 284$ & - \\
\hline (2) & $\mathbf{1 5 0} / 218 / 246$ & 138/154 & $136 / 284$ & $220 / 256$ & $248 / 256 / 284$ & - \\
\hline (2) & $\mathbf{1 5 0} / 218 / 246$ & $138 / 154$ & $136 / 284$ & $220 / 256$ & $248 / 256 / 288$ & - \\
\hline (1) & $150 / 202 / 242$ & $138 / 154$ & $136 / 284$ & $220 / 256$ & $248 / 256 / 284$ & - \\
\hline (1) & $150 / 214 / 222$ & $134 / 154$ & $136 / 284$ & $220 / 256$ & $248 / 256 / 284$ & - \\
\hline (1) & $150 / 218 / 226$ & 134/154 & $136 / 284$ & $220 / 256$ & $248 / 256 / 288$ & - \\
\hline (1) & $150 / 222 / 250$ & $138 / 154$ & $136 / 284$ & $220 / 256$ & $248 / 256 / 288$ & - \\
\hline (1) & $150 / 222 / 238$ & $138 / 154$ & $136 / 284$ & $220 / 256$ & $248 / 256 / 288$ & - \\
\hline (1) & $150 / 222 / 238$ & $134 / 154$ & $136 / 284$ & $220 / 256$ & $256 / 284$ & - \\
\hline (1) & $150 / 222 / 238$ & $134 / 154$ & 284 & $220 / 256$ & $248 / 256 / 284$ & - \\
\hline (1) & $150 / 222 / 242$ & $134 / 154$ & $136 / 284$ & $220 / 256$ & $248 / 256 / 284$ & - \\
\hline (1) & $150 / 222 / 242$ & $134 / 154$ & $136 / 284$ & 256 & $248 / 256 / 288$ & - \\
\hline (1) & $150 / 222 / 250$ & $138 / 154$ & $136 / 284$ & $220 / 256$ & $248 / 256 / 292$ & - \\
\hline (1) & $150 / 226 / 242$ & $134 / 154$ & 284 & $220 / 256$ & $248 / 256 / 288$ & - \\
\hline \multicolumn{7}{|c|}{ MMF LJJB } \\
\hline (1) & 150/198/246 & $138 / 154 / 202$ & $80 / 136 / 284$ & $220 / 256$ & $224 / 248 / 256 / 288$ & 204 \\
\hline (1) & $150 / 214 / 218$ & $138 / 154 / \overline{170}$ & $\underline{\overline{80}} / 136 / 284$ & $220 / 256$ & $\underline{224} / 248 / 256 / 284$ & - \\
\hline (1) & $150 / 218 / 242$ & $138 / 15 \overline{4}$ & $\overline{80} / 136 / 284$ & - & $\overline{224} / 248 / 256 / 284$ & 196 \\
\hline (1) & $150 / 218 / 242$ & $134 / 154 / 202$ & $\overline{80} / 136 / 284$ & $220 / 256$ & $\underline{208} / 248 / 256 / 284$ & $\underline{200}$ \\
\hline (1) & $\mathbf{1 5 0} / 218 / 246$ & $138 / 154 / \overline{174}$ & $\overline{80} / 136 / 284$ & $220 / 256$ & $\overline{224} / 248 / 256 / 288$ & $\overline{200}$ \\
\hline (1) & $150 / 222 / 238$ & $134 / 154 / \overline{158}$ & $\overline{80} / 136 / 284$ & - & $\overline{224} / 248 / 256 / 284$ & $\overline{200}$ \\
\hline (1) & $150 / 222 / 238$ & $134 / 154 / \overline{202}$ & $\overline{80} / 136 / 284$ & $220 / 256$ & $\overline{208} / 248 / 256 / 284$ & $\overline{196}$ \\
\hline (1) & $150 / 222 / 238$ & $134 / 154 / \overline{202}$ & $\overline{80} / 136 / 284$ & $220 / 256$ & $\overline{224} / 248 / 256 / 284$ & $\overline{-}$ \\
\hline (1) & $150 / 222 / 242$ & $134 / 154 / \overline{202}$ & $\overline{80} / 136 / 284$ & - & $\underline{208} / 248 / 256 / 288$ & 200 \\
\hline (1) & $150 / 222 / 246$ & $134 / 138 / \overline{154}$ & $\overline{80} / 136 / 284$ & $220 / 256$ & $\overline{224} / 248 / 256 / 288$ & $\overline{204}$ \\
\hline (1) & $150 / 222 / 250$ & $138 / 154 / \overline{202}$ & $\overline{80} / 136 / 284$ & $220 / 256$ & $\underline{224} / 248 / 256 / 292$ & $\overline{204}$ \\
\hline (1) & $150 / 222 / 250$ & $138 / 154 / \overline{214}$ & $\overline{80} / 136 / 284$ & $220 / 256$ & $\overline{224} / 248 / 256 / 288$ & $\overline{204}$ \\
\hline (1) & $150 / 226 / 242$ & $134 / 154 / \overline{202}$ & $\overline{80} / 136 / 284$ & $220 / 256$ & $\underline{224} / 248 / 256 / 288$ & $\underline{\overline{200}}$ \\
\hline
\end{tabular}

MMF A. barbouri

\begin{tabular}{|c|c|c|}
\hline đ(1) & - & $134 / 158$ \\
\hline o (1) & - & $\overline{158 / 174}$ \\
\hline ơ (1) & - & $134 / 17$ \\
\hline (1) & - & $134 / 170$ \\
\hline (1) & $186 / 370$ & $\overline{134 / 202}$ \\
\hline (1) & $186 / 370$ & $\overline{134 / 178}$ \\
\hline
\end{tabular}

Fort Ancient A. jeffersonianum

$\begin{array}{lcc}(1) & 194 / 226 & 138 \\ (1) & 206 / 222 & 146 / 154 \\ (1) & 210 / 214 & 134 / 150 \\ (1) & 210 / 222 & 134 / 150 \\ (1) & 210 / 234 & 146 / 154 \\ (1) & 210 / 238 & 134 / 146 \\ (1) & 222 / 238 & 130 / 146 \\ (1) & 230 / 242 & 138 / 146 \\ \text { o }(1) & 230 / 270 & 134 / 138\end{array}$

$\begin{array}{ll}\frac{80}{80} & - \\ \frac{80}{80} & - \\ \frac{80}{80} & -\end{array}$

$\frac{\frac{208 / 224}{224}}{\frac{208 / 224}{\frac{224}{208}}}$

$\frac{\frac{204}{204}}{\frac{204}{200}}$

Fort Ancient Unisexual

(1) $\quad 150 / 230 / 238$

$134 / 154$

$\begin{array}{cc}136 & 224 / 272 \\ 136 / 192 & 208 / 268 \\ 180 & 236 / 244 \\ 180 & 236 / 244 \\ 180 / 192 & 204 / 248 \\ 172 / 188 & 244 \\ 180 & 208 / 272 \\ 184 & 208 / 276 \\ 180 & 208 / 236\end{array}$
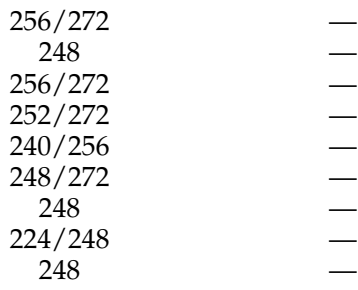

Genotypes include Ambystoma laterale (LL), A. jeffersonianum (JJ) and A. barbouri (BB). LJJB would be a tetraploid salamander having one A. laterale genome, two A. jeffersonianum genomes and one A. barbouri genome. The numbers of individuals that have the same multilocus genotype are in parentheses. Alleles known to be from A. laterale are in bold. Alleles found in tetraploid unisexuals that were also found in A. barbouri are underlined. Primers for AjeD378 only amplified A. jeffersonianum microsatellite alleles. Primers for Atex74 only amplified A. barbouri alleles. Ploidy is determined by the greatest number of alleles at any locus. No amplification of microsatellite DNA at a locus is It is assumed that most individuals are females but three $A$. barbouri individuals and one Fort Ancient $A$. jeffersonianum individual were verified as being male ( 
GenBank Acc. no. EF036597) from Montgomery County, Ohio (see Bogart et al., 2007). The $m t$ DNA sequences of unisexual individuals were identical to the haplotype found in the major unisexual clade B (compare GenBank Acc. no. EF184192). Clade B comprises eight different unisexual biotypes that are found over the range of unisexual Ambystoma (see Bogart et al., 2007). Thirteen adult individuals were tetraploid A. laterale -2 jeffersonianum - barbouri (LJJB; Table 1). The 95 triploid LJJ unisexuals had 23 different multilocus microsatellite genotypes that mostly resulted from polymorphic microsatellite DNA alleles at loci AjeD94 and AjeD422. All of the tetraploids and the diploid A. barbouri were found to have different multilocus genotypes. Only two of the seven $A$. barbouri had microsatellite DNA alleles that amplified with primers for AjeD94. They were both heterozygous AjeD94 ${ }^{186 / 370}$. Neither of these alleles, nor other alleles in this size range were found in any other individual. A. laterale microsatellite DNA alleles AjeD94 ${ }^{150}$ and AjeD346 ${ }^{284}$ (Julian et al., 2003) were found in all triploid and tetraploid unisexual individuals (Table 1, in bold). A few LJJ unisexual individuals demonstrated an unexpected loss of $A$. jeffersonianum microsatellite DNA alleles for AjeD346 and AjeD378. With the exception of these few 'missing' microsatellite DNA alleles, triploid (LJJ) and tetraploid (LJJB) unisexual individuals from MMF had the same AjeD346 $6^{136 / 284}$ and AjeD378220/256 genotypes. Most microsatellite DNA alleles found in diploid $A$. barbouri collected at MMF were also found in LJJB tetraploids from the same pond (underlined in Table 1). Representative triploid LJJ, tetraploid LJJB and diploid A. barbouri individuals from MMF are shown in Figure 1.

All the samples collected at FA had different multilocus genotypes (Table 1). None of the samples had microsatellite DNA alleles that were found in A. barbouri from MMF. Using the same microsatellite DNA criteria, 9 of the 10 samples were determined as A. jeffersonianum. One was a triploid unisexual LJJ that had the same A. laterale microsatellite DNA alleles AjeD94 ${ }^{150}$ and AjeD346 ${ }^{284}$ that the unisexuals had in MMF. Some other microsatellite DNA alleles, such as $A$. jeffersonianum alleles AjeD94230, and AjeD378 ${ }^{224}$ found in FA A. jeffersonianum and the single LJJ at FA, were not found in MMF but many alleles found in A. jeffersonianum at FA were also found in unisexuals at MMF.

Egg masses from Mitchell Memorial Forest

Many of the eggs collected from the pond did not develop to hatched larvae and no viable embryos were obtained from one egg mass (EM 1). The microsatellite DNA genotypes for offspring from the other nine egg masses (EM 2-10) are provided in Table 2. Most larvae were determined as triploid LJJ unisexuals but tetraploid LJJB individuals were found among the LJJ offspring from seven of the nine egg masses. LJJB larvae were not found in eggs that developed from EM 9 that had three surviving LJJ larvae. The two surviving larvae from EM 6 were determined as a tetraploid LJJB and a pentaploid LJJBB. Two other pentaploid LJJBB larvae were identified among the 23 larvae that hatched from EM 5. Most of the additional microsatellite alleles in tetraploid and pentaploid larva were also found in adult $A$. barbouri (underlined in Tables 1 and 2). Although many LJJ larvae from

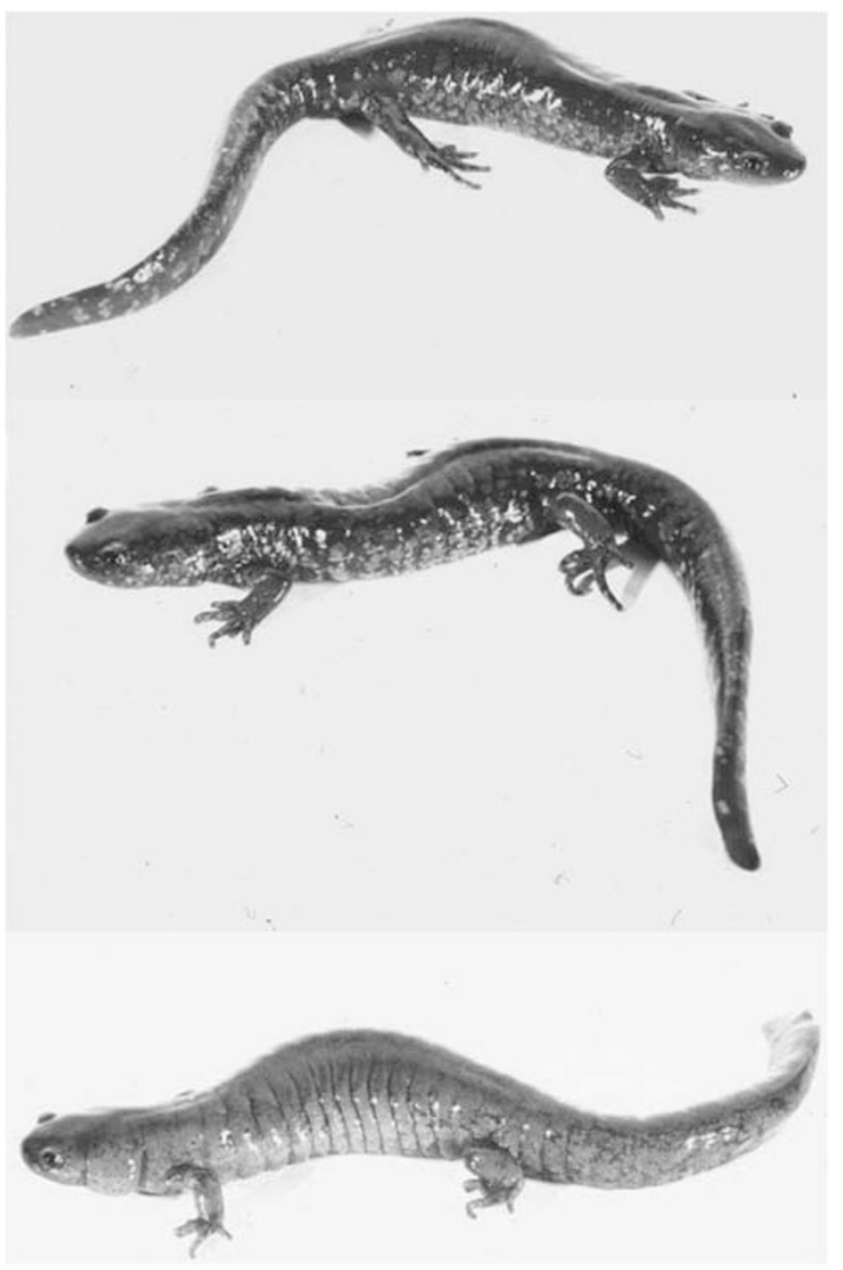

Figure 1 Representative salamanders, found in the Mitchell Memorial Forest (MMF) pond, that have been deposited in the amphibian collection in the Cleveland Museum of Natural History (CMNH). Ambystoma laterale -2 jeffersonianum (LJJ) CMNH 11790 (top), A. laterale -2 jeffersonianum - barbouri (LJJB) CMNH 11792 (middle) and A. barbouri CMNH 11791 (bottom).

the same egg masses had the same multilocus genotypes (Table 2, numbers in parentheses), LJJ larvae from EM 3, 4, 5 and 7 were determined to have two or more multilocus genotypes. Two LJJB larvae from EM 3 had the same genotype but all the other tetraploid and the pentaploid larvae had unique genotypes. Only $30.5 \%$ of the eggs hatched, and the frequency of tetraploid and pentaploid larvae was higher than in the adult sample. These data are compared in Table 3.

\section{Chromosomes}

Chromosomes were obtained from representative, viable larvae from each egg mass, which included all of the multilocus larval genotypes. Species of Ambystoma have a haploid (n) complement of 14 chromosomes. The chromosome numbers of MMF larvae $(3 n=42,4 n=56$ and $5 n=70$ ) were consistent with the expected ploidy determinations obtained by assessing ploidy with the microsatellite DNA alleles. One aneutriploid $(3 n=43)$ larva hatched from EM 9 that had three, rather than two A. jeffersonianum number 12 chromosomes and there was 
Table 2 Genotypes found at six microsatellite loci in unisexual larvae from egg masses collected from a vernal pond in Mitchell Memorial Forest (MMF), Hamilton County, Ohio

\begin{tabular}{|c|c|c|c|c|c|c|}
\hline \multirow[t]{2}{*}{ Genotype } & \multicolumn{6}{|c|}{ Microsatellite locus } \\
\hline & AjeD94 & AjeD283 & AjeD346 & AjeD378 & AjeD422 & Atex 74 \\
\hline \multicolumn{7}{|l|}{ Egg Mass 2} \\
\hline LJJ (3) & $150 / 222 / 250$ & $138 / 154$ & $136 / 284$ & $220 / 256$ & $248 / 256 / 288$ & - \\
\hline LJJB (1) & $150 / 222 / 250$ & $134 / 138 / 154$ & $\underline{80^{\mathrm{a}}} / 136 / 284$ & $220 / 256$ & $224^{\mathrm{a}} / 248 / 256 / 288$ & $\underline{200}^{\mathrm{a}}$ \\
\hline \multicolumn{7}{|l|}{ Egg Mass 3} \\
\hline LJJ (5) & $150 / 222 / 250$ & 138/154 & $136 / 284$ & $220 / 256$ & $244 / 256 / 284$ & - \\
\hline LJJB (2) & $150 / 222 / 250$ & 138/154/198 & $\underline{80}^{\mathrm{a}} / 136 / 284$ & $220 / 256$ & $224^{\mathrm{a}} / 244 / 256 / 284$ & $\underline{200}^{\mathrm{a}}$ \\
\hline LJJB (1) & $150 / 222 / 250$ & $138 / 154 / \overline{166}$ & $\overline{80}^{\mathrm{a}} / 136 / 284$ & $220 / 256$ & $\overline{224}^{\mathrm{a}} / 244 / 256 / 284$ & $\overline{192}$ \\
\hline LJJ (1) & $150 / 226 / 242$ & $134 / 15 \overline{4}$ & $-136 / 284$ & $220 / 256$ & $244 / 256 / 284$ & - \\
\hline LJJB (1) & $150 / 226 / 242$ & $134 / 154 / \underline{166}$ & $\underline{80^{\mathrm{a}}} / 136 / 284$ & $220 / 256$ & $208^{\mathrm{a}} / 244 / 256 / 284$ & $\underline{200}^{\mathrm{a}}$ \\
\hline \multicolumn{7}{|l|}{ Egg Mass 4} \\
\hline LJJ (3) & $150 / 222 / 246$ & $138 / 154$ & $136 / 284$ & $220 / 256$ & $248 / 256 / 284$ & $\overline{-}$ \\
\hline LJJB (1) & $150 / 222 / 246$ & $138 / 154 / 174^{\mathrm{a}}$ & $80^{a} / 136 / 284$ & $220 / 256$ & $224^{\mathrm{a}} / 248 / 256 / 284$ & $200^{\mathrm{a}}$ \\
\hline LJJB (1) & $150 / 226 / 242$ & $134 / 154 / \overline{174}^{\mathrm{a}}$ & $\overline{80}^{\mathrm{a}} / 136 / 284$ & $220 / 256$ & $\overline{224}^{\mathrm{a}} / 248 / 256 / 284$ & $\overline{200}^{a}$ \\
\hline \multicolumn{7}{|l|}{ Egg Mass 5} \\
\hline LJJ (12) & $150 / 222 / 246$ & $138 / 154$ & $136 / 284$ & $220 / 256$ & $244 / 256 / 284$ & - \\
\hline LJJ (2) & $150 / 226 / 242$ & $134 / 154$ & $136 / 284$ & $220 / 256$ & $248 / 256 / 280$ & - \\
\hline LJJB (1) & $150 / 222 / 246$ & $138 / 154 / 202^{\mathrm{a}}$ & $80^{a} / 136 / 284$ & $220 / 256$ & $208^{\mathrm{a}} / 248 / 256 / 284$ & 192 \\
\hline LJJB (1) & $150 / 222 / 246$ & $138 / 154 \overline{202}^{\mathrm{a}}$ & $\overline{80}^{a} / 136 / 284$ & $220 / 256$ & $\overline{224}^{\mathrm{a}} / 248 / 256 / 284$ & $\overline{192}$ \\
\hline LJJB (1) & $150 / 222 / 246$ & $1 3 4 / 1 3 8 \longdiv { 1 5 4 }$ & $\overline{80}^{\mathrm{a}} / 136 / 284$ & $220 / 256$ & $208^{\mathrm{a}} / 248256 / 284$ & $\overline{192}$ \\
\hline LJJB (1) & $150 / 226 / 242$ & 134/154/166 & $\overline{80}^{\mathrm{a}} / 136 / 284$ & $220 / 256$ & $2 \overline{208^{a}} / 248 / 256 / 284$ & $\overline{192}$ \\
\hline LJJB (1) & $150 / 226 / 242$ & $134 / 154 / 202^{\mathrm{a}}$ & $\overline{80}^{\mathrm{a}} / 136 / 284$ & $220 / 256$ & $\overline{224}^{\mathrm{a}} / 248 / 256 / 284$ & $\overline{200^{a}}$ \\
\hline LJJB (1) & $150 / 226 / 242$ & $134 / 154 \overline{202}^{\mathrm{a}}$ & $80^{a} / 284$ & $220 / 256$ & $\overline{224}^{\mathrm{a}} / 248 / 256 / 284$ & $\overline{200}^{\mathrm{a}}$ \\
\hline LJJB (1) & $150 / 226 / 242$ & $134 / 154 / \overline{202}^{\mathrm{a}}$ & $80^{\mathrm{a}} / 136 / 284$ & $220 / 256$ & $\overline{224}^{\mathrm{a}} / 248 / 256 / 284$ & 二 \\
\hline LJJBB (1) & $150 / 226 / 242$ & $134 / 154 / 16 \overline{6 / 174^{a}}$ & $\overline{80}^{\mathrm{a}} / 136 / 284$ & $220 / 256$ & $208^{\mathrm{a}} / 224^{\mathrm{a}} / 248 / 256 / 284$ & $192 / 200^{\mathrm{a}}$ \\
\hline LJJBB (1) & $226 / 242$ & $134 / 15 \overline{4 / 1} \underline{\underline{66}}$ & $\overline{80}^{\mathrm{a}} / 136 / 284$ & $220 / 256$ & $208^{\mathrm{a} / 2} 48 / 256 / 284$ & $\overline{192} / \overline{200}^{\mathrm{a}}$ \\
\hline \multicolumn{7}{|l|}{ Egg Mass 6} \\
\hline $\begin{array}{l}\text { LJJBB (1) } \\
\text { LJIB (1) }\end{array}$ & $\mathbf{1 5 0 / 2 2 6 / 2 4 6}$ & $134 / 154 / 166 / 202^{\mathrm{a}}$ & $80^{a} / 136 / 284$ & $220 / 256$ & $208^{\mathrm{a}} / 224^{\mathrm{a}} / 248 / 256 / 284$ & $\frac{200^{a}}{20 a^{a}}$ \\
\hline LJJB (1) & $150 / 226 / 246$ & $134 / 15 \overline{4 / 20 \overline{2}^{\mathrm{a}}}$ & $\underline{80}^{a} / 136 / 284$ & $220 / 256$ & $\underline{208^{\mathrm{a}}} / 248 / 256 / 284$ & $\underline{200}^{\mathrm{a}}$ \\
\hline \multicolumn{7}{|l|}{ Egg Mass 7} \\
\hline LJJ (6) & $\mathbf{1 5 0} / 218 / 226$ & $134 / 154$ & $136 / 284$ & $220 / 256$ & $248 / 256 / 284$ & - \\
\hline LJJ (2) & $150 / 218 / 222$ & $134 / 154$ & $136 / 284$ & $220 / 256$ & $248 / 256 / 284$ & - \\
\hline LJJ (2) & $150 / 222 / 242$ & $134 / 154$ & $136 / 284$ & $220 / 256$ & $248 / 256 / 284$ & - \\
\hline LJJ (5) & $150 / 222 / 246$ & $134 / 154$ & $136 / 284$ & $220 / 256$ & $248 / 256 / 284$ & - \\
\hline LJJ (5) & $150 / 222 / 250$ & $134 / 154$ & $136 / 284$ & $220 / 256$ & $248 / 256 / 284$ & - \\
\hline LJJB (1) & $150 / 222 / 246$ & $134 / 154 / \underline{178}^{\mathrm{a}}$ & $\underline{80}^{\mathrm{a}} / 136 / 284$ & $220 / 256$ & $\underline{208}^{\mathrm{a}} / 248256 / 284$ & $\underline{196}^{\mathrm{a}}$ \\
\hline \multicolumn{7}{|l|}{ Egg Mass 8} \\
\hline LJJB (1) & $150 / 226 / 242$ & $134 / 154$ & $80^{\mathrm{a}} / 284$ & $220 / 256$ & $208^{\mathrm{a}} / 248 / 256 / 284$ & $200^{\mathrm{a}}$ \\
\hline \multicolumn{7}{|l|}{ Egg Mass 9} \\
\hline LJJ (3) & $150 / 222 / 246$ & $138 / 154$ & $136 / 284$ & $220 / 256$ & $248 / 256 / 284$ & - \\
\hline \multicolumn{7}{|l|}{ Egg Mass 10} \\
\hline LJJ (19) & 150/218/246 & $138 / 154$ & $136 / 284$ & $220 / 256$ & $248 / 256 / 284$ & - \\
\hline LJJB (1) & $150 / 218 / 242$ & $138 / 154 / 182$ & $80^{\mathrm{a}} / 136 / 284$ & $220 / 256$ & $224^{\mathrm{a}} / 248 / 256 / 284$ & $\underline{200}^{\mathrm{a}}$ \\
\hline LJJB (1) & $150 / 218 / 250$ & $138 / 15 \overline{4}$ & $\overline{80}^{\mathrm{a}} / 136 / 284$ & $220 / 256$ & $\overline{224}^{\mathrm{a}} / 248 / 256 / 284$ & $\overline{200}^{a}$ \\
\hline LJJB (1) & $150 / 218 / 246$ & $138 / 154 / \underline{190}$ & $8^{a} / 136 / 284$ & $220 / 256$ & $224^{\mathrm{a}} / 248 / 256 / 284$ & $1^{196}{ }^{\mathrm{a}}$ \\
\hline
\end{tabular}

Nuclear genomes include Ambystoma laterale (L), A. jeffersonianum (J) and A. barbouri (B). The number of larvae within an egg mass that have the same multilocus genotype are in parentheses. Alleles known to be from A. laterale are in bold. Alleles assumed to be from A. barbouri are underlined. Primers for AjeD378 only amplified A. jeffersonianum microsatellite alleles. Primers for Atex74 only amplified A. barbouri alleles. Ploidy was determined by the greatest number of alleles at any locus or from the minimum number of possible genomes. LJJBB would be a pentaploid larva having one A. laterale genome, two A. jeffersonianum genomes and two A. barbouri genomes. No amplification of microsatellite DNA alleles at a locus is -

aAlleles with the same mobility were found in adult specimens of A. barbouri from the same pond (see Table 1).

also a small exchanged $A$. jeffersonianum segment on the long arm of A. laterale chromosome 10. Bi et al. (2007b) described the karyotype of this unique individual. GISH performed on several LJJ larvae verified that there were 14 A. laterale chromosomes and 28 A. jeffersonianum chromosomes. Three-way GISH applied to tetraploid LJJB spreads revealed 14 A. laterale chromosomes, 28 A. jeffersonianum chromosomes and 14 A. barbouri chromosomes (Figure 2). Pentaploid individuals were found to have $28 \mathrm{~A}$. barbouri chromosomes. 
Table 3 Number of eggs in each egg mass, viability of embryos and frequency of microsatellite DNA genotypes from larvae compared with 115 adult salamanders collected in a vernal pond in Mitchell Memorial Forest (MMF), Hamilton County, Ohio.

\begin{tabular}{lcccccc}
\hline Egg mass & Eggs $(\mathrm{n})$ & \multicolumn{5}{c}{ Genotype } \\
\cline { 3 - 7 } & & Hatched & LJJ & LJJB & LJJBB & $B B$ \\
\hline 1 & 13 & 0 & 0 & 0 & 0 & 0 \\
2 & 15 & 4 & 3 & 1 & 0 & 0 \\
3 & 20 & 10 & 6 & 4 & 0 & 0 \\
4 & 52 & 15 & 13 & 2 & 0 & 0 \\
5 & 63 & 23 & 14 & 7 & 2 & 0 \\
6 & 14 & 2 & 0 & 1 & 1 & 0 \\
7 & 70 & 21 & 20 & 1 & 0 & 0 \\
8 & 8 & 5 & 4 & 1 & 0 & 0 \\
9 & 14 & 3 & 3 & 0 & 0 & 0 \\
10 & 75 & 22 & 19 & 3 & 0 & 0 \\
Total & 344 & 105 & 82 & 20 & 3 & 0 \\
$\%(105)$ & & 30.5 & 78.1 & 19.0 & 2.8 & 0 \\
Adults & & & 95 & 13 & 0 & 7 \\
$\%$ (115) & & & 82.6 & 11.3 & 0 & 6.1 \\
\hline
\end{tabular}

Genomes include Ambystoma laterale (L), A. jeffersonianum (J) and A. barbouri (B).

The percentages provided for the four genotypes found among the larvae from the egg masses are calculated from 105 hatched larvae.

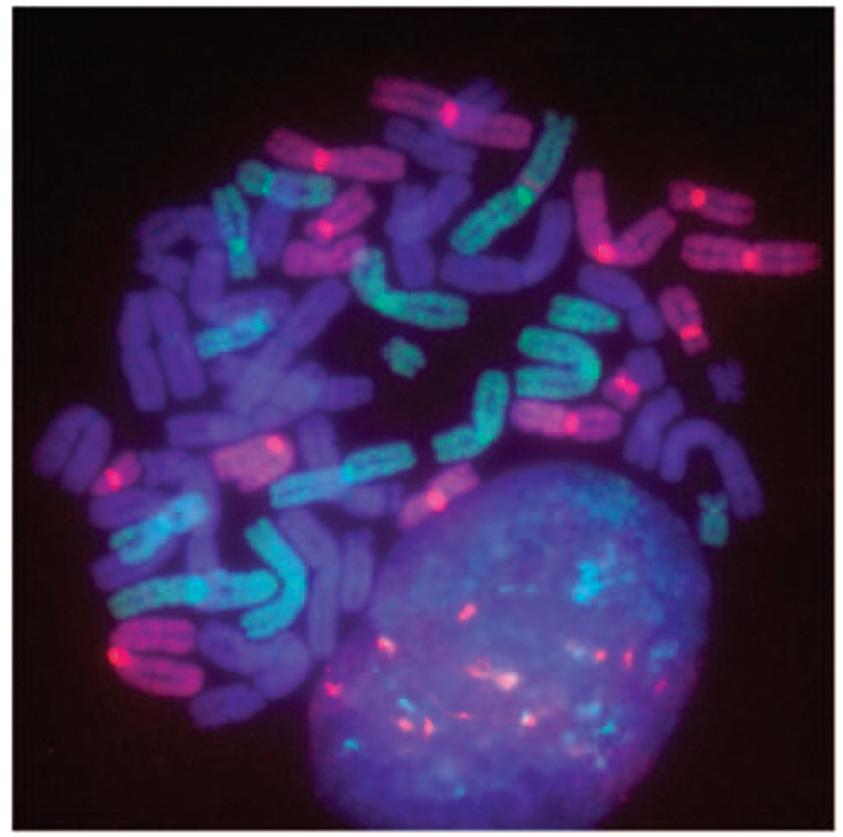

Figure 2 Fluorescence genomic in situ hybridization (GISH) applied to a chromosome squash preparation of a tetraploid Ambystoma laterale -2 jeffersonianum - barbouri (LJJB) larva from Mitchell Memorial Forest (MMF). A. laterale chromosomes (14) are green, A. jeffersonianum chromosomes (28) are blue and A. barbouri chromosomes (14) are pink. The sphere at the bottom of the figure is an interphase nucleus.

\section{Discussion}

\section{Ambystoma in Mitchell Memorial Forest pond}

Our data are consistent with a population of LJJ unisexual individuals that successfully exist and are being maintained by using $A$. barbouri as a sperm donor in MMF. A. jeffersonianum, the expected sperm donor for LJJ unisexuals (Bogart and Klemens, 1997, 2008), was not found among 115 adults collected from the pond but $6 \mathrm{~A}$. barbouri were found and 3 were determined as males. Sex was not confirmed for most adult individuals that were subsequently released after collecting tail tip samples. The same microsatellite DNA alleles that were found in our sample of adult $A$. barbouri were also found in tetraploid LJJB adult individuals (underlined alleles in Table 1) and in tetraploid LJJB and pentaploid LJJBB larvae (underlined alleles in Table 2). Most larvae were the product of gynogenetic reproduction, where the A. barbouri sperm was not incorporated. Sperm was incorporated in many developing embryos which gave rise to tetraploid LJJB individuals that most likely can survive to maturity based on finding 13 LJJB adult tetraploids in this pond. One egg mass (EM 6; Tables 2 and 3) may have been laid by a tetraploid LJJB female because the only eggs that developed were an LJJB tetraploid that was possibly a gynogenetic offspring, and an LJJBB pentaploid that was the result of ploidy elevation. Tetraploid LJJB and pentaploid LJJBB larvae were also found among offspring from EM 5 but most larvae from that egg mass were triploid LJJ (Table 3). If the female that produced EM 5 was LJJB, the A. barbouri genome must have been eliminated during oogenesis in half of the developing eggs. If the female was LJJ, the ploidy-elevated pentaploids must have incorporated two A. barbouri genomes. Physiological polyspermy is a known feature of fertilization in salamanders, a characteristic which distinguishes those amphibians from monospermatic frogs, but mechanisms normally exist within the egg cytoplasm that prohibit the fusion of more than one principal sperm nucleus with the egg nucleus (Elinson, 1986). Thus, it is unlikely that multiple sperm nuclei are incorporated in single eggs. Gynogenesis is a known and common event in the development of many unisexual oocytes (Elinson et al., 1992; Bogart et al., 1989, 2007). The fact that all seven tetraploids, and the two pentaploid larvae from EM 5 have different multilocus genotypes, poses an additional problem if we assume that the tetraploids from those eggs were gynogenetic offspring. The most plausible explanation is that all the eggs in EM 5 were not laid by the same female and the pentaploid individuals were derived from eggs laid by a tetraploid female. Although we selected concise egg masses that were separated from other masses when the eggs were collected, we could not confirm that any egg mass was laid by one specific female. The different microsatellite DNA multilocus genotypes of some larvae from the same egg mass support the possibility that some egg masses included eggs from more than one female. Data from the egg masses (Table 2) and from other studies (Bogart and Licht, 1986; Bogart et al., 1987, 1989, 2007) demonstrate that tetraploids arise from triploid females through sperm incorporation in unreduced eggs. Such ploidy elevation events provide important information concerning the identity of a sperm donor as can rare pentaploids that are presumed to be derived from tetraploid females (Lowcock and Murphy, 1991; Phillips et al., 1997). None of the larvae were $A$. barbouri and we cannot confirm that $A$. barbouri actually reproduce bisexually in the MMF pond. A. barbouri is reported to lay eggs in cryptic sites and under rocks in streams (Petranka, 1998). We searched likely A. barbouri 
egg deposition sites in the MMF pond but only egg masses attached to branches in the water column were found. Sampling larvae later in the season might answer this question.

\section{Microsatellite DNA alleles}

Unisexual salamanders provide an interesting system to examine mutational events that can include microsatellite DNA alleles and other sequence-based changes in the nuclear genome. The A. laterale genome that is present in all unisexuals is maintained in lineages that are isolated from bisexual individuals of $A$. laterale, so genome exchange of $A$. laterale genomes is not possible (Bi et al., $2008 b)$. Unisexuals in such populations have a single A. laterale genome, so meiotic recombinations that involve $A$. laterale chromosomes would be intergenomic, and can be observed using GISH. When unisexuals engage in genomic exchange with a sperm donor, genomes and the microsatellite DNA alleles they contain can be gained or lost. If genome exchange does not occur between a sperm donor and unisexual individuals or is very rare, microsatellite DNA alleles and the frequency of those alleles would be expected to deviate over time only through mutational events and genetic drift, which would result in alleles that were not shared among sympatric bisexual and unisexual individuals. Thus, microsatellite DNA alleles provide evidence that can support or reject genome replacement. When genome replacement does occur, the frequency of such occurrence can be estimated using comparative data derived from putative gene donors. Evidence for genome replacement is found in FA. The single unisexual LJJ found at FA shared most of its microsatellite DNA alleles with sympatric $A$. jeffersonianum (Table 1 ) and some of those alleles were not found in MMF LJJ unisexuals.

With the exception of one pentaploid larva from EM 5 that was missing AjeD94 ${ }^{150}$, all of the unisexuals sampled from MMF and FA had the same $A$. laterale microsatellite DNA alleles AjeD94 ${ }^{150}$ and AjeD346 ${ }^{284}$, which is consistent with previous observations that all unisexuals have at least one A. laterale genome (Bogart, 2003). The monomorphic nature of $A$. laterale alleles for these, normally highly polymorphic, loci likely relates to the fact that the closest populations of $A$. laterale are in northwestern Ohio and northern Indiana (Petranka, 1998), so there is no possibility that an A. laterale genome could be exchanged in southern Ohio unisexuals as it is in populations that have A. laterale male sperm donors (Bi et al., 2008b). As all MMF unisexuals possess AjeD284 ${ }^{154}$ and AjeD422 256 alleles, these could also be A. laterale alleles but these alleles were found in A. jeffersonianum individuals from $\mathrm{FA}$ and the single unisexual LJJ from FA did not possess AjeD422 256 . Primers for microsatellite DNA locus AjeD378 only amplified $A$. jeffersonianum alleles. Only two alleles (AjeD378220 and AjeD378256) were found in the unisexuals from MMF where all but one individual had heterozygous 220/256 genotypes (Tables 1 and 2). That one individual was either a 256/256 homozygote or the AjeD378 $8^{220}$ allele failed to amplify because of some unknown mutational event. Neither of these AjeD378 alleles was found in $A$. jeffersonianum individuals or the LJJ unisexual at FA. The loss of A. jeffersonianum AjeD346 ${ }^{136}$ microsatellite DNA alleles in one LJJB
Table 4 A comparison of the number of microsatellite DNA alleles found at five loci in Ambystoma laterale -2 jeffersonianum (LJJ) unisexual salamanders from Mitchell Memorial Forest (MMF) compared with LJJ unisexuals from a population in southern Ontario (Bogart et al., 2007; Sudden Tract), and with A. jeffersonianum individuals from Fort Ancient (FA; present study)

$\mathrm{n} \quad$ Microsatellite locus Total

AjeD94 AjeD238 AjeD346 AjeD378 AjeD422

\begin{tabular}{lrllrlrl}
\hline MMF (LJJ) & 115 & 11 & 4 & 2 & 2 & 5 & 25 \\
Ontario (LJJ) & 16 & 17 & 7 & 17 & 8 & 13 & 62 \\
FA (JJ) & 9 & 11 & 7 & 6 & 9 & 6 & 39 \\
\hline
\end{tabular}

offspring from EM 5, all five offspring of EM 8 (Table 2) and two adult LJJ unisexuals (Table 1) is more difficult to explain because this allele is expected to be homozygous for $A$. jeffersonianum genomes in triploid LJJ, tetraploid LJJB and pentaploid LJJBB unisexuals. A mutational event on the primer region that might prohibit amplification in one genome would not be expected to affect more than one $A$. jeffersonianum genome. That allele is present in a presumed homozygous AjeD346 ${ }^{136 / 136}$ and a heterozygous AjeD346 $6^{136 / 192}$ condition in A. jeffersonianum individuals from FA (Table 1). The MMF unisexuals have a reduced allelic richness compared with LJJ individuals in a southern Ontario population and A. jeffersonianum at FA (Table 4). The monomorphic A. laterale alleles contribute to the reduced polymorphism but there are fewer $A$. jeffersonianum alleles as well. We found only two A. jeffersonianum AjeD378 alleles in the MMF pond. These alleles were not found in any sampled FA individual. Based on our data, the JJ genomes probably persist in isolation, and only in unisexuals, in the pond at MMF in a similar manner to the $A$. laterale genome (above) but, unlike $A$. laterale, $\mathrm{MMF}$ is in the known range of $A$. jeffersonianum (Petranka, 1998).

\section{Embryonic mortality}

Only $30.5 \%$ of the eggs hatched (Table 3), but embryonic mortality is typically associated with unisexual salamanders and this average hatching rate is higher than observations made for other unisexual populations such as those on Kelleys Island, Ohio (19.5\%; Bogart et al., 1987) and Pelee Island, Ontario (21.4\%; Bogart and Licht, 1986). The hatching success varied among the egg masses from 0 (EM 1) to 62.5\% (EM 8; Table 3). Embryonic mortality has been linked to intergenomic recombinations and translocations (Bi and Bogart, 2006; Bi et al., 2007a, 2007b) during female meiosis and other possible complexities in the reproductive system used by unisexuals. A small exchanged segment on $A$. laterale chromosome 10 (see $\mathrm{Bi}$ et al., 2007b) was present in all three larvae that hatched from EM 9 but the expected reciprocal exchanged segment was not found in A. jeffersonianum chromosomes. We speculate that the A. jeffersonianum chromosome that possessed this A. laterale exchanged segment was replaced with a normal A. jeffersonianum chromosome when, historically, $A$. jeffersonianum was a sperm donor. In the absence of an A. laterale sperm donor, all descendants of the original female that possessed that chromosome mutation would maintain the small exchanged segment. The selective 
advantages or disadvantages of intergenomic translocations and recombinations are not known. Such mutational events are common in many populations (Bi et al., 2008a) but were not observed in MMF larvae from every egg mass. Comparing the sampled adults and larvae (Table 3), pentaploids were only found among the larvae, and there were more tetraploid larvae than we found in our adult sample. Based on our data, and other studies (Lowcock et al., 1991; Phillips et al., 1997), tetraploid and pentaploid unisexuals have a reduced fitness compared with triploid individuals.

\section{Persistence of unisexual salamanders}

Unisexual kleptogens depend on males of bisexual species for recruitment. The stolen sperm is used to initiate cleavage and may or may not be incorporated in developing eggs to elevate the ploidy level or to replace a genome (Bogart et al., 1989; Elinson et al., 1992; Bi et al., 2008b). Unisexuals in MMF use $A$. barbouri as a sperm donor and the barbouri genome is included in tetraploid (LJJB) and pentaploid (LJJBB) individuals but we did not observe possible genomic exchange that would have been evidenced by finding LJB or LBB or BJJ triploid individuals. This was an unexpected observation. In their examination of more than 2000 A. laterale, A. jeffersonianum and unisexuals from 216 sites (Bogart and Klemens, 1997, 2008), the associations of unisexuals and sperm donating males are very clear. There is a direct correlation such that LLJ are sympatric with and use $A$. laterale as a sperm donor, and LJJ use sympatric A. jeffersonianum. Also, where A. texanum (TT) is used as a sperm donor, triploid (LTT) unisexuals are found, and where $A$. tigrinum (TiTi) is used as a sperm donor, triploid LTTi unisexuals exist. We interpret these observations as evidence that support genome replacement of genomes in unisexual individuals with genomes from these other sympatric sperm donors. A similar situation to that found in MMF is believed to exist in some LJJ populations in Illinois and central Indiana where $A$. jeffersonianum has not been found and $A$. texanum is believed to serve as a sperm donor (Spolsky et al., 1992, Phillips et al., 1997). Tetraploid LJJT that are capable of producing pentaploids were also found (Phillips et al., 1997). Both studies assume that all of the triploid unisexuals are LJJ clones and that there is no evidence for genome replacement but we question the identification of the triploids used in those studies. Spolsky et al. (1992) identified unisexual individuals by morphology (snout-vent length vs internasal distance) and allozyme electrophoretic mobility for the isozyme locus superoxide dismutase (SOD-1). A. jeffersonianum, $A$. laterale and $A$. texanum have all been shown to be homozygous for SOD-1 allozymes and individuals for each species demonstrate a distinctly different and easily separated mobility in the unisexuals (Bogart et al., 1985, 1987). Spolsky et al. (1992) found two different SOD electrophoretic phenotypes for LJJ individuals (50/50/100 and 50/75/100) and claimed that both the 50 and 75 allozymes were $A$. jeffersonianum alleles. These data are unexpected if gynogenesis is the only reproductive method used by all LJJ individuals and show that LJJ populations in Illinois and Indiana cannot be the same genetic clone. Genome replacement would be a possible explanation if $A$. jeffersonianum in some unknown population were polymorphic for SOD-1 allozymes. It is also possible that the SOD 50/50/100 individuals are LJJ and the 50/75/100 individuals are LTJ, which would be expected if $A$. texanum were to replace $A$. jeffersonianum as a sperm donor. No genetic markers were used by Phillips et al. (1997). They examined the morphology and ploidy of individuals and, using those criteria, determined that all the triploids were LJJ, the tetraploids were LJJT and pentaploids were LJJTT. It would be interesting and important to revisit populations in Illinois and Indiana using microsatellite DNA loci and GISH.

The 23 different LJJ genotypes (Table 1) suggest that $A$. jeffersonianum was most likely a sperm donor in the MMF pond and was recently extirpated. Adult and larva LJJB individuals demonstrate that $A$. barbouri has served as a sperm donor in the pond for several years. If rare male A. jeffersonianum still exist in the pond we expect to find a few LJJJ unisexuals, which are found in low frequencies in other ponds where A. jeffersonianum exists with LJJ unisexuals (Bogart et al., 2007; Bogart and Klemens, 1997, 2008). Even though $A$. barbouri genomes have been incorporated in tetraploid and pentaploid individuals, we did not find genome replacing BJJ, LBJ or LBB unisexuals in the MMF pond. Perhaps these genome combinations are not viable or are less fit compared with LJJ and LJJB combinations. As the unisexual $m t$ DNA sequences are most similar to $A$. barbouri, we expected that the barbouri genome would be readily accepted and incorporated in reduced unisexual eggs. We suggest that time and natural selection could be factors that enable successful genome replacement. It may require some lengthy period of isolation where unisexuals exist with the 'wrong' sperm donor before genome replacement is initially accomplished and, once accomplished, new genome combinations can spread through the population. The MMF pond is fairly typical A. jeffersonianum habitat and would be considered marginal $A$. barbouri habitat based on ecological criteria provided by Petranka (1998). In MMF, unisexuals that possessed one or two barbouri genomes (LJB, LBB, BJJ) might be expected to have a reduced fitness compared with LJJ unisexuals in that habitat.

\section{Concluding remarks}

A. barbouri is the fifth known species that can serve as a sperm donor to enable unisexual Ambystoma to persist. In keeping with unisexuals that use other sperm donors, A. barbouri sperm is incorporated in unreduced unisexual eggs to elevate the ploidy to tetraploid and pentaploid levels. But, unlike unisexuals that use the other sperm donors, no triploids were found that have an $A$. barbouri genome. Apparently, unisexual kleptogens steal A. barbouri sperm for gynogenetic reproduction and, incidentally, for ploidy elevation. This Ohio population demonstrates the evolutionary significance and amazing flexibility of kleptogenesis. If one sperm donor is rendered unavailable through extinction or habitat alteration, another species can substitute as a sperm donor, so the geographical range of unisexuals is only limited by the ranges of acceptable sperm donors. As all unisexuals have a mitochondrial DNA that is most similar to $A$. barbouri and very different from any of the other four sperm donors, we expected that genome 
replacement would be facilitated and, perhaps, an $A$. barbouri genome could even replace the $A$. laterale genome that is found in all unisexuals. We found no evidence for such genome replacement. As most unisexuals had an LJJ genotype, we expected that A. jeffersonianum was present in this Ohio population and could possibly hybridize with $A$. barbouri to mimic an original hybridization event that used an $A$. barbourilike female and gave rise to unisexual Ambystoma. A. jeffersonianum was not found in the pond and we did not detect any possible $A$. barbouri $\times A$. jeffersonianum hybrids. All the unisexuals possessed an $A$. laterale genome, which eliminates this possibility. We believe that we have observed an interesting aspect of kleptogenesis where unisexuals are relying on a new sperm donor for recruitment in the absence of a former contributor. Genome replacement may not be successful at this time because of current selective advantages for unisexuals that maintain two $A$. jeffersonianum genomes (for example, LJJ, LJJB) in the present environment.

We now know that genomes from at least five distinct species can be incorporated in various unisexual diploid or polyploid biotypes. Additionally, individual unisexuals can produce genetically variable offspring through intergenomic translocations and recombinations even when a donor's sperm is only used to initiate development of unreduced eggs and is not incorporated. These salamanders are not the only unisexuals that have the capability to exploit genomes from sympatric males to escape potential clonal demise. Manning et al. (2005) described sperm stealing in lizards. Aspidocelis neomexicana, a parthenogenetic diploid species, can rarely cross with $A$. sexlineatus viridis males and produce triploid lizards. A triploid parthenogenetic species, A. neotessalatus, resulted from the diploid parthenogenetic species A. tessellatus and one or more hybridizations of that species with $A$. sexlineatus viridis. Triploid hybrids are also produced when Aspidocelis tessellatus crosses with A. tigris marmorata. In fishes, unisexual hybrids of mummichogs (Fundulus heteroclitus) and banded killifish (F. diaphanus) are found on the coast of Nova Scotia in Eastern Canada. Based on microsatellite alleles, Hernandez Chavez and Turgeon (2007) observed multiple clones of the unisexuals, which led these authors to speculate that there have been several independent origins of unisexuality resulting from hybridization of these two species of fish or that the unisexual hybrids may not be completely gynogenetic. Despite clonal variability, all clones share the same unique $F$. diaphanus $m t$ DNA haplotype. Schories et al. (2007) used microsatellite alleles to document clonal diversity in the gynogenetic Amazon molly (Poecilia formosa). Microsatellite alleles were shared in diploids and triploids that were attributed to paternal genomic introgression events of sperm donors into triploid lineages. The planarian flatworm (Schmidtea polychroa) is a sperm-dependent simultaneous hermaphrodite that is incapable of self fertilization (D'Souza et al., 2006). In gynogenetic flatworms, sperm stimulates development and usually is not incorporated in the offspring. D'Souza et al. (2006) made crosses between parthenogens as well as between sexual and parthenogenetic flatworms. Using microsatellites, they found that $12 \%$ of the females' offspring were the result of partial genetic exchange that resulted in tetraploids derived from gynogenetic triploid flatworms. Flatworm genomes can increase from $3 n$ to $4 n$ and they can also decrease from $4 n$ to $3 n$. Genetic exchange can occur within and between ploidy levels.

These, and other, studies use molecular genomic techniques to improve our understanding of genetic variation in 'clonal' female organisms and to document intergenomic interactions with males. It is evident that unisexual organisms need not be doomed by a lack of genetic variability. Mechanisms have independently evolved for female unisexuals to capture and to use genomes from foreign donors. These facts defy any speculations or generalizations that unisexuals lack genetic variation, and thus cannot evolutionarily compete with organisms that engage in 'normal' bisexual reproduction. In addition to the obvious questions concerning the significance of sexual reproduction and assortative mating, unisexual organisms can provide answers to many fundamental questions in genetics, ecology and evolution. The consequences of intergenomic interaction and the independent evolution of unisexual organisms are poorly understood phenomena. Rather than being complex, unisexuals do not fit very comfortably with our expectations. The perceived genetic and evolutionary complexities associated with unisexuality are probably derived from inadequate study but are more likely to be derived from the perception that 'normal' bisexual reproduction drives evolution.

\section{Acknowledgements}

Animals were collected and processed under permits issued by the Ohio Department of Natural Resources. Access to Mitchell Memorial Forest was provided by the Hamilton County Park District and to Fort Ancient by the Ohio Historical Society. Animal use was approved by permits issued by Wright State University Laboratory Animal Care and Use Committee and the Animal Care Committee of the University of Guelph who approved our protocol for larval experimentation. The Ohio Environmental Protection Agency provided valuable technical assistance. We also thank B Husband for assistance with the fluorescent microscope and J Fu for supporting our laboratory work. Our research was supported by a Systematics Research Fund grant from the Linnean Society to JB and a discovery grant from the National Science and Engineering Research Council, Canada (NSERC) to JPB.

\section{References}

Avise JC (2008). Clonality: The Genetics, Ecology, and Evolution of Sexual Abstinence in Vertebrate Animals. Oxford University Press: New York.

Bi K, Bogart JP (2006). Identification of intergenomic recombinations in unisexual slamanaders of the genus Ambystoma by genomic in situ hybridization. Cytogenet Genome Res 112: 307-312.

Bi K, Bogart JP, Fu J (2007a). Intergenomic translocations in unisexual salamanders of the genus Ambystoma (Amphibia, Caudata). Cytogenet Genome Res 116: 289-297.

Bi K, Bogart JP, Fu J (2007b). Two rare aneutriploids in the unisexual Ambystoma (Amphibia, Caudata) identified by GISH indicating two different types of meiotic errors. Cytogenet Genome Res 119: 127-130. 
Bi K, Bogart JP, Fu J (2008a). Genealogical relationships of southern Ontario polyploid unisexual salamanders (genus Ambystoma) inferred from intergenomic exchanges and major rDNA cytotypes. Chromosome Res 16: 275-289.

Bi K, Bogart JP, Fu J (2008b). The prevalence of genome replacement in unisexual salamanders of the genus Ambystoma (Amphibia, Caudata) revealed by nuclear gene genealogy. BMC Evol Biol 8: 158.

Bogart JP (2003). Genetics and systematics of hybrid species. In: Sever DM (ed) Reproductive Biology and Phylogeny of Urodela, vol. 1. M/s Science: Enfield, NH. pp 109-134.

Bogart JP, Bi K, Fu J, Noble DWA, Niedzwiecki J (2007). Unisexual salamanders (genus Ambystoma) present a new reproductive mode for eukaryotes. Genome 50: 119-136.

Bogart JP, Elinson RP, Licht LE (1989). Temperature and sperm incorporation in polyploid salamanders. Science 246: 1032-1034.

Bogart JP, Klemens MW (1997). Hybrids and genetic interactions of mole salamanders (Ambystoma jeffersonianum and A. laterale) (Amphibia: Caudata) in New York and New England. Am Mus Nat Hist Novitates 3218: 1-78.

Bogart JP, Klemens MW (2008). Additional distributional records of Ambystoma laterale, A. jeffersonianum (Amphibia: Caudata) and their unisexual kleptogens in northeastern North America. Am Mus Nat Hist Novitates 3627: 1-58.

Bogart JP, Licht LE (1986). Reproduction and the origin of polyploids in hybrid salamanders of the genus Ambystoma. Can J Genet Cytol 28: 605-617.

Bogart JP, Licht LE, Oldham MJ, Darbyshire SJ (1985). Electrophoretic identification of Ambystoma laterale and Ambystoma texanum as well as their diploid and triploid interspecific hybrids (Amphibia: Caudata) on Pelee Island, Ontario. Can J Zool 63: 340-347.

Bogart JP, Lowcock LA, Zeyl CW, Mable BK (1987). Genome constitution and reproductive biology of the Ambystoma hybrid salamanders on Kelleys Island in Lake Erie. Can I Zool 65: 2188-2201.

Dawley RM, Bogart JP (1989). Evolution and Ecology of Unisexual Vertebrates. New York State Museum Bulletin 466.

Dessauer HC, Cole CJ (1989). Diversity between and within nominal forms of unisexual teiid lizards. In: Dawley RM, Bogart JP (eds). Evolution and Ecology of Unisexual Vertebrates New York State Museum Bulletin 466; pp 49-71.

Downs FL (1978). Unisexual Ambystoma from the Bass Islands of Lake Erie. Occas Pap Mus Zool Univ Mich 685: 1-36.

D'Souza TG, Storhas M, Schulenberg H, Beukeboom LW, Michiels NK (2004). Occasional sex in an 'asexual' polyploid hermaphrodite. Proc Royal Soc London B 271: 1001-1007.

D'Souza TG, Schulte RD, Schulenburg H, Michiels NK (2006). Paternal inheritance in parthenogenetic forms of the planarian Schmidtea polychroa. Heredity 97: 97-101.

Elinson RP (1986). Fertilization in amphibians: the ancestry of the block to polyspermy. Int Rev Cytol 101: 59-100.

Elinson RP, Bogart JP, Licht LE, Lowcock LA (1992). Gynogenetic mechanisms in polyploid hybrid salamanders. $J$ Exp Zool 264: 93-99.

Hernandez Chavez C, Turgeon J (2007). Asexual and sexual hybrids between Fundulus diaphanous and F.heteroclitus in the Canadian Atlantic region. Mol Ecol 16: 1467-1480.

Julian SE, King TL, Savage WK (2003). Novel Jefferson salamander, Ambystoma jeffersonianum, microsatellite DNA markers detect population structure and hybrid complexes. Mol Ecol Notes 3: 95-97.

Kraus F (1985a). Unisexual salamander lineages in northwestern Ohio and southeastern Michigan: a study of the consequences of hybridization. Copeia 1985: 309-324.

Kraus F (1985b). A new unisexual salamander from Ohio. Occas Pap Mus Zool Univ Mich 709: 1-24.

Lowcock LA, Griffith AH, Murphy RW (1991). Size in relation to sex, hybridity, ploidy, and breeding dynamics in central Ontario populations of the Ambystoma laterale-jeffersonianum complex. J Herpetol 26: 46-53.

Lowcock LA, Murphy RW (1991). Pentaploidy in hybrid salamanders demonstrates enhanced tolerance of multiple chromosome sets. Experientia 47: 490-493.

Manning GT, Cole CJ, Dessauer HC, Walker JM (2005). Hybridization between parthenogentic lizards (Aspidoscelis neomexicana) and gonochoristic lizards (Aspidoscelis sexlineata viridis) in New Mexico: ecological, morphological, cytological, and molecular context. Am Mus Nat Hist Novitates 3429: 1-56.

Maynard Smith J (1992). Age and the unisexual lineage. Nature 356: $661-662$

Morris MA (1985). A hybrid Ambystoma platineum x A. tigrinum from Indiana. Herpetologica 41: 267-271.

Morris MA, Brandon RA (1984). Gynogenesis and hybridization between Ambystoma platineum and Ambystoma texanum in Illinois. Copeia 1984: 324-337.

Niedzwiecki J (2005). Evolutionary history and hybridization of two mole salamanders sister species from different habitats. PhD Thesis, University of Kentucky.

Normark BB, Judson OP, Moran NA (2003). Genomic signatures of ancient asexual lineages. Biol J Linn Soc 79: 69-84.

Petranka JW (1998). Salamanders of the United States and Canada. Smithsonian Instution Press: Washington and London.

Phillips CA, Uzzell T, Spolsky CM, Serb JM, Szafoni RE, Pollowy TR (1997). Persistent high levels of tetraploidy in salamanders of the Ambystoma jeffersonianum complex. J Herpetol 31: 530-535.

Schories S, Lampert KP, Lamatsch DK, Garcia de León FJ, Schartl M (2007). Analysis of a possible independent origin of triploid $P$. formosa outside of the Rió Purificación river system. Front Zool 4: 13.

Shaffer HB, McKnight ML (1996). The polytypic species revisited: genetic differentiation and molecular phylogenetics of the tiger salamander Ambystoma tigrinum (Amphibia: Caudata) complex. Evolution 50: 417-433.

Spolsky C, Phillips CA, Uzzell T (1992). Gynogenetic reproduction in hybrid mole salamanders (genus Ambystoma). Evolution 46: 1935-1944.

Thompson JD, Gibson TJ, Plewniak F, Jeanmougin F, Higgins DG (1994). The CLUSTAL X windows interface: flexible strategies for multiple sequence alignment aided by quality anlaysis tools. Nucleic Acids Res 25: 4876-4882.

Uzzell T (1964). Relations of the diploid and triploid species of the Ambystoma jeffersonianum complex (Amphibia, Caudata). Copeia 1964: 257-300.

Williams GR (1975). Sex and Evolution. Princeton University Press: Princeton, NJ.

Williams RN, Dewoody JA (2004). Fluorescent dUTP helps characterize 10 novel tetranucleotide microsatellites from an enriched salamander (Ambystoma texanum) genomic library. Mol Ecol Notes 4: 17-19. 